\title{
A HOMOTOPY THEORETIC REALIZATION OF STRING TOPOLOGY
}

\author{
RALPH L. COHEN AND JOHN D.S. JONES
}

\begin{abstract}
Let $M$ be a closed, oriented manifold of dimension $d$. Let $L M$ be the space of smooth loops in $M$. In [2] Chas and Sullivan defined a product on the homology $H_{*}(L M)$ of degree $-d$. They then investigated other structure that this product induces, including a Batalin -Vilkovisky structure, and a Lie algebra structure on the $S^{1}$ equivariant homology $H_{*}^{S^{1}}(L M)$. These algebraic structures, as well as others, came under the general heading of the "string topology" of $M$. In this paper we will describe a realization of the Chas - Sullivan loop product in terms of a ring spectrum structure on the Thom spectrum of a certain virtual bundle over the loop space. We also show that an operad action on the homology of the loop space discovered by Voronov has a homotopy theoretic realization on the level of Thom spectra. This is the "cactus operad" defined in [6] which is equivalent to operad of framed disks in $\mathbb{R}^{2}$. This operad action realizes the Chas - Sullivan BV structure on $H_{*}(L M)$. We then describe a cosimplicial model of this ring spectrum, and by applying the singular cochain functor to this cosimplicial spectrum we show that this ring structure can be interpreted as the cup product in the Hochschild cohomology, $H H^{*}\left(C^{*}(M) ; C^{*}(M)\right)$.
\end{abstract}

\section{INTRODUCTION}

Let $M^{d}$ be a smooth, closed $d$ - dimensional manifold, and let $L M=C^{\infty}\left(S^{1}, M\right)$ be the space of smooth loops in $M$. In [2] Chas and Sullivan described an intersection product on the homology $H_{*}(L M)$ having total degree $-d$,

$$
\circ: H_{q}(L M) \otimes H_{r}(L M) \rightarrow H_{q+r-d}(L M)
$$

In this paper we show that this product is realized by a geometric structure, not on the loop space itself, but on the Thom spectrum of a certain bundle over $L M$. We describe this structure both homotopy theoretically and simplicially, and in so doing describe the relationship of the Chas - Sullivan product to the cup product in Hochschild cohomology. We now make these statements more precise.

Date: August 23, 2018.

The first author was partially supported by a grant from the NSF . 
Consider the standard parameterization of the circle by the unit interval, exp $:[0,1] \rightarrow S^{1}$ defined by $\exp (t)=e^{2 \pi i t}$. With respect to this parameterization we can regard a loop $\gamma \in L M$ as a map $\gamma:[0,1] \rightarrow M$ with $\gamma(0)=\gamma(1)$. Consider the evaluation map

$$
\begin{aligned}
e v: L M & \rightarrow M \\
\gamma & \rightarrow \gamma(1) .
\end{aligned}
$$

Now let $\iota: M \rightarrow \mathbb{R}^{N+d}$ be a fixed embedding of $M$ into codimension $N$ Euclidean space. Let $\nu^{N} \rightarrow M$ be the $N$ - dimensional normal bundle. Let $T h\left(\nu^{N}\right)$ be the Thom space of this bundle. Recall the famous result of Atiyah [1] that $T h\left(\nu^{N}\right)$ is Spanier - Whitehead dual to $M$ with a disjoint basepoint which we denote by $M_{+}$. Said more precisely, let $M^{-T M}$ be the spectrum given by desuspending this Thom space,

$$
M^{-T M}=\Sigma^{-(N+d)} T h\left(\nu^{N}\right) .
$$

Then there are maps of spectra

$$
S^{0} \rightarrow M_{+} \wedge M^{-T M} \text { and } M_{+} \wedge M^{-T M} \rightarrow S^{0}
$$

that establish $M^{-T M}$ as the $S-d u a l$ of $M_{+}$. That is, these maps induce an equivalence with the function spectrum $M^{-T M} \simeq \operatorname{Map}\left(M_{+}, S^{0}\right)$.

Notice by duality, the diagonal map $\Delta: M \rightarrow M \times M$ induces a map of spectra

$$
\Delta^{*}: M^{-T M} \wedge M^{-T M} \rightarrow M^{-T M}
$$

that makes $M^{-T M}$ into a ring spectrum with unit. The unit $S^{0} \rightarrow M^{-T M}$ is the map dual to the projection $M_{+} \rightarrow S^{0}$.

Now let $T h\left(e v^{*}\left(\nu^{N}\right)\right)$ be the Thom space of the pull back bundle $e v^{*}\left(\nu^{N}\right) \rightarrow L M$. Define the spectrum

$$
L M^{-T M}=\Sigma^{-(N+d)} T h\left(e v^{*}\left(\nu^{N}\right)\right) .
$$

The main goal of this paper is to define and study a product structure on the spectrum $L M^{-T M}$ which among other properties makes the evaluation map $e v: L M^{-T M} \rightarrow M^{-T M}$ a map of ring spectra. Here, by abuse of notation, ev is referring the map of Thom spectra induced by the evaluation map ev $L M \rightarrow M$. We will prove the following theorem.

Theorem 1. Let $M$ be a smooth, closed manifold of dimension d. The spectrum $L M^{-T M}$ is a ring spectrum with unit, whose multiplication

$$
\mu: L M^{-T M} \wedge L M^{-T M} \longrightarrow L M^{-T M}
$$


satisfies the following properties.

(1) The evaluation map ev: $L M^{-T M} \rightarrow M^{-T M}$ is a map of ring spectra.

(2) There is a map of ring spectra $\rho: L M^{-T M} \rightarrow \Sigma^{\infty}\left(\Omega M_{+}\right)$where the target is the suspension spectrum of the based loop space with a disjoint basepoint. Its ring structure is induced by the usual product on the based loop space. In the case when $M$ is oriented, the homology of the map $\rho_{*}$ is given by the composition

$$
\rho_{*}: H_{q}\left(L M^{-T M}\right) \stackrel{\tau}{\cong} H_{q+d} L M \stackrel{\iota}{\longrightarrow} H_{q}(\Omega M)
$$

where like above, $\tau$ is the Thom isomorphism, and the map ८ takes $a(q+d)$ cycle in LM and intersects it with the based loop space viewed as a codimension $d$ submanifold.

(3) When $M$ is orientable the ring structure is compatible with the Chas - Sullivan homology product in the sense that the following diagram commutes:

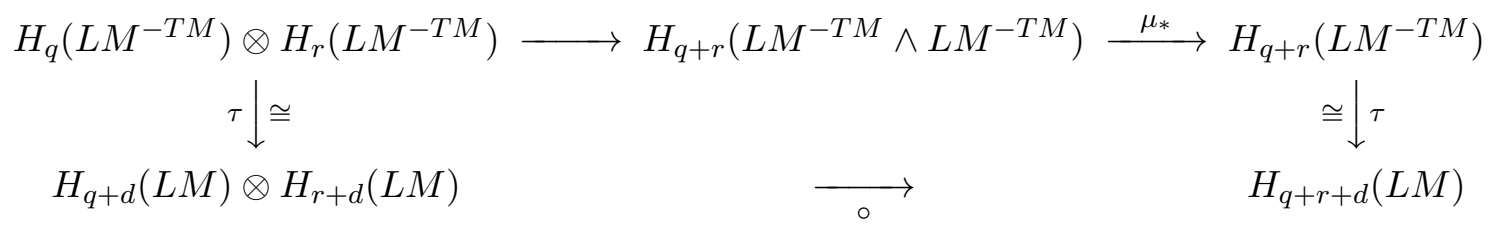

Remarks. 1. The fact that $L M^{-T M}$ is a ring spectrum was also observed by W. Dwyer and H. Miller.

2.In [2] Chas and Sullivan define a regrading of the homology of the loop space

$$
\mathbb{H}_{q}=H_{q+d}(L M)
$$

with respect to which the product $\circ$ is of total degree zero. We observe that the Thom isomorphism defines an isomorphism

$$
\mathbb{H}_{*} \cong H_{*}\left(L M^{-T M}\right)
$$

which respects gradings, and by the above theorem is an isomorphism of rings, where the ring structure on the right hand side comes from the ring spectrum structure of $L M^{-T M}$.

We also show that there is a similar homotopy theoretic realization of the operad structure on the homology of the loop space found by Voronov [6]. The operad involved is the "cactus operad" $\mathcal{C}$ which has the homotopy type of the operad of framed little disks in $\mathbb{R}^{2}$ (theorem 2.3 of [6]). Using a result of Getzler [3], Voronov showed this operad structure realizes the Batalin - Vilkovisky structure on $H_{*}(L M)$ discovered by Chas and Sullivan. Therefore the 
following theorem can be viewed as a homotopy theoretic realization of the $B V$ - algebra on $H_{*}(L M)$.

Theorem 2. Let $M$ be a smooth, closed, $d$-dimensional manifold. Then for each $k$ there is a virtual bundle $\theta_{k}$ over $\mathcal{C}_{k} \times(L M)^{k}$ of virtual dimension $-d(k-1)$, and a stable map from its Thom spectrum to the loop space,

$$
\zeta_{k}:\left(\mathcal{C}_{k} \times(L M)^{k}\right)^{\theta_{k}} \rightarrow L M
$$

that satisfies the following property. If $M$ is orientable, then an orientation on $M$ induces an orientation on the virtual bundle $\theta_{k}$, and in homology, the compostion

$$
H_{q+d(k-1)}\left(\mathcal{C}_{k} \times(L M)^{k}\right) \stackrel{\tau_{*}}{\cong} H_{q}\left(\left(\mathcal{C}_{k} \times(L M)^{k}\right)^{\theta_{k}}\right) \stackrel{\left(\zeta_{k}\right)_{*}}{\longrightarrow} H_{q}(L M)
$$

is the Voronov operad structure, which in turn realizes the Chas - Sullivan BV structure.

Our final result has to do with the simplicial structure of $L M^{-T M}$, and the resulting simplicial description of the product.

Let $S_{*}^{1}$ be the simplicial set decomposition of the circle which has one zero simplex and one nondegenerate one simplex. In this decomposition there are $n+1 n$-simplices, all of which are degenerate for $n>1$. We write this as $S_{n}^{1}=\{n+1\}$. Now given any space $X$, there is a resulting cosimplicial model, $\mathbb{X}_{*}$, for the free loop space $L X$. The $n$ - simplices of $\mathbb{X}_{*}$ are given by maps

$$
\mathbb{X}_{n}=\operatorname{Map}\left(S_{n}^{1}, X\right)=\operatorname{Map}(\{n+1\}, X)=X^{n+1} .
$$

The coface and codegeneracy maps of $(L X)_{*}$ are dual to the face and degeneracy maps of $S_{*}^{1}$.

Our next result states that there is a similar cosimplicial model for $L M^{-T M}$.

Theorem 3. For $M$ a closed, oriented manifold, the spectrum $L M^{-T M}$ has the structure of a cosimplicial spectrum which we write as $\left(\mathbb{L}_{M}\right)_{*}$. The $k$ simplices of $\left(\mathbb{L}_{M}\right)_{*}$ are given by

$$
\left(\mathbb{L}_{M}\right)_{k}=\left(M^{k}\right)_{+} \wedge M^{-T M} .
$$

This cosimplicial structure has the following properties.

(1) The ring structure of $L M^{-T M}$ is realized on the (co)simplicial level by pairings

$$
\mu_{k}:\left(\left(M^{k}\right)_{+} \wedge M^{-T M}\right) \wedge\left(\left(M^{r}\right)_{+} \wedge M^{-T M}\right) \rightarrow\left(M^{k+r}\right)_{+} \wedge M^{-T M}
$$

defined by

$$
\mu_{k}\left(x_{1}, \cdots, x_{k} ; u\right) \wedge\left(y_{1}, \cdots, y_{r} ; v\right)=\left(x_{1}, \cdots, x_{k}, y_{1}, \cdots y_{r} ; \Delta^{*}(u \wedge v)\right)
$$


where $\Delta^{*}$ is the ring structure defined on $M^{-T M}$ described earlier.

(2) If $M$ is orientable, then applying the singular chain functor $C_{*}(-)$ to the cosimplicial space $\left(\mathbb{L}_{M}\right)_{*}$ yields a natural chain homotopy equivalence between the chains of $L M^{-T M}$ and the Hochschild cochain complex

$$
f_{*}: C_{*}\left(L M^{-T M}\right) \cong C_{*}\left(\left(\mathbb{L}_{M}\right)_{*}\right) \cong C H^{*}\left(C^{*}(M) ; C^{*}(M)\right) .
$$

Here the notation $C H^{*}(A ; A)$ refers to the Hochschild cochain complex of the form

$$
A \rightarrow \operatorname{Hom}(A ; A) \rightarrow \cdots \rightarrow \operatorname{Hom}\left(A^{\otimes n} ; A\right) \rightarrow \operatorname{Hom}\left(A^{\otimes n+1} ; A\right) \rightarrow \cdots
$$

Furthermore, the pairing on the chains $C_{*}\left(L M^{-T M}\right)$ induced by the ring spectrum structure corresponds via the chain homotopy equivalence $f_{*}$ to the cup product pairing in $C H^{*}\left(C^{*}(M) ; C^{*}(M)\right)$. This gives ring isomorphisms in homology,

$$
\mathbb{H}_{*} \cong H_{*}\left(L M^{-T M}\right) \underset{f_{*}}{\cong} H H^{*}\left(C^{*}(M) ; C^{*}(M)\right) .
$$

Remark. The fact that the Chas - Sullivan product is realized as the cup product in Hochschild cohomology was also observed by T. Tradler, and will appear in his CUNY Ph.D thesis.

The paper will be organized as follows. In section 1 we will show how to realize the Chas - Sullivan product using the Pontrjagin - Thom constuction. We will use this to prove theorem 1. In section 2 we recall the definition of the cactus operad $\mathcal{C}$ and prove theorem 2. In section 3 we will recall the cosimplicial study of the loop space done by the second author in [⿶], apply the Thom spectrum construction to it, and use it to prove theorem 3.

The authors are grateful to J. Klein, I. Madsen, J. Morava, G. Segal, D. Sullivan, U. Tillmann, and A. Voronov for helpful conversations regarding this material. 


\section{The RING StRUCture on $L M^{-T M}$ : The PROOF OF TheOREM 1}

In this section we will describe the ring spectrum structure of the Thom spectrum $L M^{-T M}$ defined in the introduction, discuss some its properties, and prove theorem 1.

Proof. The multiplicative structure $\mu: L M^{-T M} \wedge L M^{-T M} \rightarrow L M^{-T M}$ will be defined using the Pontrjagin - Thom construction. We therefore begin by recalling some properties of this construction.

Let

$$
e: P^{k} \hookrightarrow N^{n+k}
$$

be an embedding of closed manifolds. Let $\nu_{e}$ be a tubular neighborhood of $e\left(P^{k}\right)$, which we identify with the total space of the normal bundle of the embedding. Let

$$
\tau: N^{n+k} \rightarrow \nu_{e} \cup \infty
$$

be the Pontrjagin - Thom collapse map to the one point compactification, defined by

$$
\tau(x)= \begin{cases}x & \text { if } x \in \nu_{e} \\ \infty & \text { if } x \notin \nu_{e} .\end{cases}
$$

If we identify the compactification with the Thom space of the normal bundle, $\nu_{e} \cup \infty \cong$ $P^{\nu_{e}}$, then in the oriented case we can apply the Thom isomorphism $u_{*}: H_{q}\left(P^{\nu_{e}}\right) \stackrel{\cong}{\longrightarrow} H_{q-n}\left(P^{k}\right)$, to get the "push-forward", or "umkehr" map,

$$
e_{!}: H_{q}\left(N^{k}\right) \stackrel{\tau_{*}}{\longrightarrow} H_{q}\left(P^{\nu_{e}}\right) \stackrel{u_{*}}{\cong} H_{q-n}\left(P^{k}\right) .
$$

Recall that in the case of the diagonal embedding of a $d$-dimensional closed manifold,

$$
\Delta: M^{d} \rightarrow M^{d} \times M^{d}
$$

that the normal bundle is isomorphic to the tangent bundle,

$$
\nu_{\Delta} \cong T M
$$

so that the Pontrjagin - Thom map is a map $\tau: M \times M \rightarrow M^{T M}$. Furthermore, in the oriented case the push - forward map in homology,

$$
\Delta_{!}: H_{*}\left(M^{d} \times M^{d}\right) \stackrel{\tau_{*}}{\longrightarrow} H_{*}\left(M^{T M}\right) \stackrel{u_{*}}{\cong} H_{*-d}\left(M^{d}\right) .
$$

is simply the intersection product.

Now the Pontrjagin - Thom construction also applies when one has a vector bundle over the ambient manifold of an embedding. That is, if one has an embedding $e: P^{k} \hookrightarrow N^{n+k}$ 
as above, and if one has a vector bundle (or virtual bundle) $\zeta \rightarrow N$, then one obtains a Pontrjagin - Thom map

$$
\tau: \zeta \cup \infty \rightarrow \nu\left(\iota^{*}(\zeta)\right) \cup \infty
$$

where $\nu\left(\iota^{*}(\zeta)\right)$ is the tubular neighborhood of the induced embedding of total spaces $\iota^{*}(\zeta) \hookrightarrow$ $\zeta$. Now $\zeta \cup \infty$ is the Thom space $N^{\zeta}$, and $\nu\left(\iota^{*}(\zeta)\right) \cup \infty$ is the Thom space $P^{\iota^{*}(\zeta) \oplus \nu_{e}}$. So the Pontrjagin - Thom map is a map

$$
\tau: N^{\zeta} \rightarrow P^{\iota^{*}(\zeta) \oplus \nu_{e}}
$$

Moreover this construction works when $\zeta$ is a virtual bundle over $N$ as well. In this case when $\zeta=-E$, where $E \rightarrow N$ is a $k$-dimensional vector bundle over $N$, then the Thom spectrum $N^{\zeta}=N^{-E}$ is defined as follows. Suppose the bundle $E$ is embedded in a $k+M$ dimensional trivial bundle, $E \hookrightarrow N \times \mathbb{R}^{k+M}$. Let $E^{\perp}$ be the $M$ - dimensional orthogonal complement bundle to this embedding $E^{\perp} \rightarrow N$. Then

$$
N^{-E}=\Sigma^{-(N+k)} N^{E^{\perp}}
$$

Notice that when $E$ is oriented, the Thom isomorphism is of the form $u_{*}: H^{q}(N) \cong$ $H^{q-k}\left(N^{-E}\right)$.

In particular, applying the Pontrjagin - Thom construction to the diagonal embedding $\Delta: M \hookrightarrow M \times M$, using the virtual bundle $-T M \times-T M$ over $M \times M$, we get a map of Thom spectra,

$$
\tau:(M \times M)^{-T M \times-T M} \rightarrow M^{T M \oplus \Delta^{*}(-T M \times-T M)}
$$

or,

$$
\tau: M^{-T M} \wedge M^{-T M} \rightarrow M^{-T M}
$$

When $M$ is oriented, this map still realizes the intersection pairing on $H_{*}(M)$ after applying the Thom isomorphism. The map $\tau$ defines a ring spectrum structure on $M^{-T M}$ that is well known to be the Spanier - Whitehead dual of the diagonal map $\Delta: M \rightarrow M \times M$.

To construct the ring spectrum pairing $\mu: L M^{-T M} \wedge L M^{-T M} \rightarrow L M^{-T M}$, we basically "pull back" the structure $\tau$ over the loop space. 
To make this precise, let $e v \times e v: L M \times L M \rightarrow M \times M$ be the product of the evaluation maps, and define $L M \times_{M} L M$ to be the fiber product, or pull back:

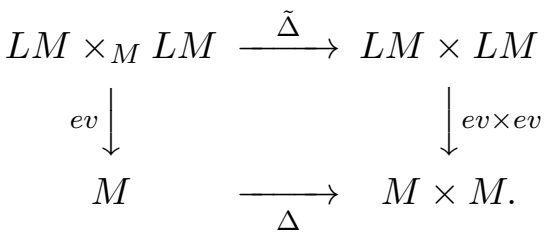

Notice that $L M \times_{M} L M$ is a codimension $d$ submanifold of the infinite dimensional manifold $L M \times L M$, and can be thought of as

$$
L M \times_{M} L M=\{(\alpha, \beta) \in L M \times L M \text { such that } \alpha(0)=\beta(0)\} .
$$

Notice that there is also a natural map from $L M \times_{M} L M$ to the loop space $L M$ defined by first applying $\alpha$ and then $\beta$. That is,

$$
\begin{aligned}
\gamma: L M \times_{M} L M & \rightarrow L M \\
(\alpha, \beta) & \rightarrow \alpha * \beta
\end{aligned}
$$

where

$$
\alpha * \beta(t)= \begin{cases}\alpha(2 t) & \text { if } 0 \leq t \leq \frac{1}{2} \\ \beta(2 t-1) & \text { if } \frac{1}{2} \leq t \leq 1 .\end{cases}
$$

Notice that when restricted to the product of the based loop spaces, $\Omega M \times \Omega M \subset L M \times M$ $L M$, then $\gamma$ is just the $H$ - space product on the based loop space, $\Omega M \times \Omega M \rightarrow \Omega M$.

Remark. This definition needs to be modified slightly since $\alpha * \beta$ may not be smooth at $1 / 2$ or 1 . This is dealt with in a standard way by first "dampening" $\alpha$ and $\beta$ by using a smooth bijection $\phi:[0,1] \rightarrow[0,1]$ with the property that all of its derivatives approach zero at the endpoints $\{0\}$ and $\{1\}$, to reparameterize $\alpha$ and $\beta$. This will allow them to be "spliced" together by the above formula without losing any smoothness. This is a standard construction, so we leave its details to the reader.

Notice that by its definition (1.1) the embedding $\tilde{\Delta}: L M \times_{M} L M \hookrightarrow L M \times L M$ has a tubular neighborhood $\nu(\tilde{\Delta})$ defined to be the inverse image of the tubular neighborhood of the diagonal $\Delta: M \hookrightarrow M \times M$ :

$$
\nu(\tilde{\Delta})=e v^{-1}(\nu(\Delta)) .
$$

Therefore this neighborhood is homeomorphic to the total space of the $d$ - dimensional vector bundle given by pulling back the normal bundle of the embedding $\Delta$, which is the 
tangent bundle of $M$ :

$$
\nu(\tilde{\Delta}) \cong e v^{*}\left(\nu_{\Delta}\right)=e v^{*}(T M) .
$$

Thus there is a Pontrjagin - Thom collapse map

$$
\tau: L M \times L M \rightarrow L M \times_{M} L M^{e v^{*}(T M)} .
$$

As described earlier, we ease the notation by refering to this Thom spectrum as $(L M \times M$ $L M)^{T M}$. By the naturality of the Pontrjagin - Thom construction, we have a commutative diagram of spectra,

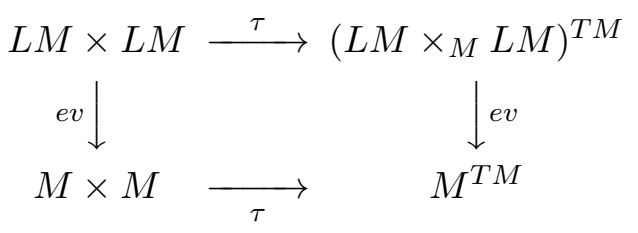

Since in the oriented case $\tau_{*}: H_{*}(M \times M) \rightarrow H_{*}\left(M^{T M}\right) \cong H_{*-d}(M)$ is the intersection product, then

$$
H_{*}(L M \times L M) \stackrel{\tau_{*}}{\longrightarrow} H_{*}\left(\left(L M \times_{M} L M\right)^{T M}\right) \stackrel{u_{*}}{\longrightarrow} H_{*-d}\left(L M \times_{M} L M\right)
$$

can be viewed (as is done in Chas - Sullivan [2]) as taking a cycle in $L M \times L M$, and "intersecting" it with the codimension $d$ submanifold $L M \times_{M} L M$.

Now observe that the map $\gamma: L M \times_{M} L M \rightarrow L M$ defined above (1.2) preserves the evaluation map. That is, the following diagram commutes:

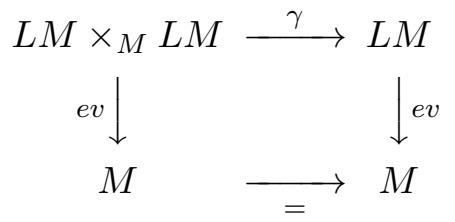

Thus $\gamma$ induces a map of bundles $\gamma: e v^{*}(T M) \rightarrow e v^{*}(T M)$, and therefore a map of Thom spectra,

$$
\gamma:\left(L M \times_{M} L M\right)^{T M} \rightarrow L M^{T M} .
$$

Now consider the composition

$$
\tilde{\mu}: L M \times L M \stackrel{\tau}{\longrightarrow}\left(L M \times_{M} L M\right)^{T M} \stackrel{\gamma}{\longrightarrow} L M^{T M}
$$

In the oriented case the homomorphism

$$
H_{*}(L M \times L M) \stackrel{\tilde{\mu}_{*}}{\longrightarrow} H_{*}\left(L M^{T M}\right) \stackrel{u_{*}}{\cong} H_{*-d}(L M)
$$


takes a cycle in $L M \times L M$, intersects in with the codimension $d$ - submanifold $L M \times{ }_{M} L M$, and maps it via $\gamma$ to $L M$. This is the definition of the Chas - Sullivan product $H_{*}(L M)$.

Now as we did before with the diagonal embedding, we can perform the Pontrjagin Thom construction when we pull back the virtual bundle $-T M \times-T M$ over $L M \times L M$. That is, we get a map of Thom spectra

$$
\tau:(L M \times L M)^{(e v \times e v)^{*}(-T M \times-T M)} \longrightarrow\left(L M \times{ }_{M} L M\right)^{e v^{*}(T M) \oplus e v^{*}\left(\Delta^{*}(-T M \times-T M)\right)} .
$$

But since $e v^{*}\left(\Delta^{*}(-T M \times-T M)\right)=e v^{*}(-2 T M)$, we have

$$
\tau: L M^{-T M} \wedge L M^{-T M} \longrightarrow\left(L M \times_{M} L M\right)^{T M \oplus-2 T M}=\left(L M \times_{M} L M\right)^{-T M} .
$$

Now by the commutativity of (1.4), $\gamma$ induces a map of Thom spectra,

$$
\gamma:\left(L M \times_{M} L M\right)^{-T M} \rightarrow L M^{-T M}
$$

and so we can define the ring structure on the Thom spectrum $L M^{-T M}$ to be the composition

$$
\mu: L M^{-T M} \wedge L M^{-T M} \stackrel{\tau}{\longrightarrow}\left(L M \times_{M} L M\right)^{-T M} \stackrel{\gamma}{\longrightarrow} L M^{-T M}
$$

A few properties of this map $\mu$ are now immediately verifiable.

First, $\mu$ is associative. This follows from the naturality of the Pontrjagin - Thom construction, and the fact that the map $\gamma$ is associative. (Strictly speaking, formula (1.2) is $A_{\infty}$ - associative as is the usual formula for the product on the based loop space, $\Omega M$. However the standard trick of replacing $\Omega M$ with "Moore loops" changes the $A_{\infty}$ structure to a strictly associative structure. The same technique applies to the map $\gamma$. Otherwise, the spectrum $L M^{-T M}$ will have the structure of an $A_{\infty}$ ring spectrum.)

Also, notice tha $L M^{-T M}$ has a unit, $\iota: S^{0} \rightarrow L M^{-T M}$, defined by the composition

$$
\iota: S^{0} \stackrel{j}{\longrightarrow} M^{-T M} \stackrel{\sigma}{\longrightarrow} L M^{-T M}
$$

where $j$ is the unit of the ring spectrum structure of $M^{-T M}$, and $\sigma$ is the map of Thom spectra induced by the section of the evaluation map $e v: L M \rightarrow M$ defined by viewing points in $M$ as constant loops.

Notice furthermore that in the oriented case, after applying the Thom isomorphism, $\mu_{*}$ induces the same homomorphism as $\tilde{\mu}_{*}$, and so by (1.6) the following diagram commutes:

$$
\begin{array}{ccc}
H_{q-2 d}\left(L M^{-T M} \wedge L M^{-T M}\right) & \stackrel{\mu_{*}}{\longrightarrow} & H_{q-2 d}\left(L M^{-T M}\right) \\
u_{*} \mid \cong & \cong \downarrow u_{*} \\
H_{q}(L M \times L M) & & \\
\longrightarrow & H_{q-d}(L M)
\end{array}
$$


where $\circ: H_{q}(L M \times L M) \rightarrow H_{q-d}(L M)$ is the Chas - Sullivan product. This proves part (3) of theorem 1 .

Now by the naturality of the Pontrjagin - Thom construction, the following diagram of Thom spectra commutes (compare (1.3))

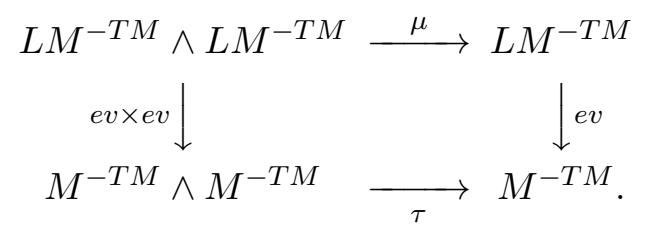

Thus the evaluation map ev $: L M^{-T M} \rightarrow M^{-T M}$ is a map of ring spectra, which proves part 1 of theorem 1 .

We now verify part 2 of theorem 1. Let $x_{0} \in M$ be a base point, and consider the following pullback diagram:

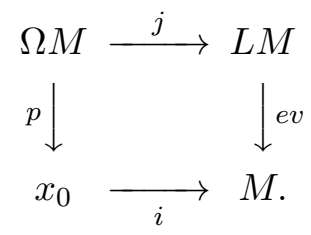

Thus the embedding $j: \Omega M \hookrightarrow L M$ is an embedding of a codimension $d$ submanifold, and has tubular neighborhood, $\nu(j)$ equal to the inverse image of the tubular neighborhood of the inclusion of the basepoint $i: x_{0} \hookrightarrow M$. This tubular neighborhood is simply a disk $D^{d}$, and so

$$
\nu(j) \cong \Omega M \times D^{d} \cong \epsilon^{d}
$$

where $\epsilon^{d}$ reflects the $d$ dimensional trivial bundle over $\Omega M$. Thus the Thom - Pontrjagin construction makes sense, and is a map

$$
\tau: L M \rightarrow \Omega M^{\epsilon^{d}}=\Sigma^{d}\left(\Omega M_{+}\right)
$$

where the last space is the $d$ - fold suspension of $\Omega M$ with a disjoint basepoint. In homology, the homomorphism

$$
\tau_{*}: H_{q}(L M) \rightarrow H_{q}\left(\Sigma^{d}\left(\Omega M_{+}\right)=H_{q-d}(\Omega M)\right.
$$

denotes the map that is obtained by intersecting a $q$-cycle in $L M$ with the codimension $d$ submanifold $\Omega M$. 
By performing the Pontrjagin - Thom construction after pulling back the virtual bundle $-T M$ over $L M$, we get a map of Thom spectra

$$
\tau: L M^{-T M} \rightarrow(\Omega M)^{\epsilon^{d} \oplus j^{*} e v^{*}(-T M)} .
$$

But by the commutativity of diagram (1.10), $j^{*} e v^{*}(-T M)=p^{*} i^{*}(-T M)$, which is the trivial, virtual $-d$ dimensional bundle which we denote $\epsilon^{-d}$. So the Pontrjagin - Thom map is therefore a map of spectra

$$
\rho: L M^{-T M} \stackrel{\tau}{\longrightarrow}(\Omega M)^{\epsilon^{d} \oplus j^{*} e v^{*}(-T M)}=(\Omega M)^{\epsilon^{d} \oplus \epsilon^{-d}}=\Sigma^{\infty}\left(\Omega M_{+}\right)
$$

where $\Sigma^{\infty}\left(\Omega M_{+}\right)$denotes the suspension spectrum of the based loop space of $M$ with a disjoint basepoint. To complete the proof of theorem 1 we need to prove that $\rho: L M^{-T M} \rightarrow$ $\Sigma^{\infty}\left(\Omega M_{+}\right)$is a map of ring spectra. Toward this end, consider the following diagram of pull back squares:

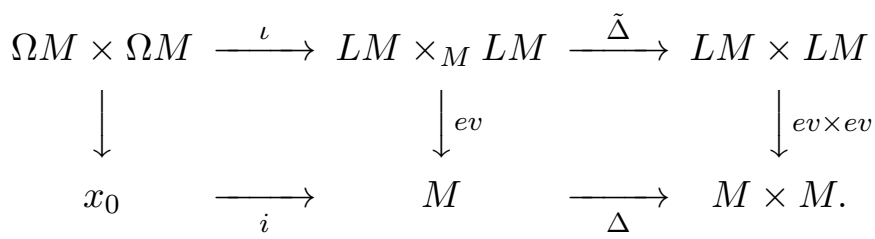

This gives Pontrjagin - Thom maps

$$
L M^{-T M} \wedge L M^{-T M} \stackrel{\tau}{\longrightarrow}\left(L M \times_{M} L M\right)^{-T M} \stackrel{\tau}{\longrightarrow} \Sigma^{\infty}\left(\Omega M_{+}\right) \wedge \Sigma^{\infty}\left(\Omega M_{+}\right) .
$$

Notice that by the naturality of the Pontrjagin - Thom construction, the above composition is equal to

$$
\rho \wedge \rho: L M^{-T M} \wedge L M^{-T M} \longrightarrow \Sigma^{\infty}\left(\Omega M_{+}\right) \wedge \Sigma^{\infty}\left(\Omega M_{+}\right)
$$

Now notice that by the formula for the map $\gamma: L M \times_{M} L M \rightarrow L M$, the following diagram commutes:

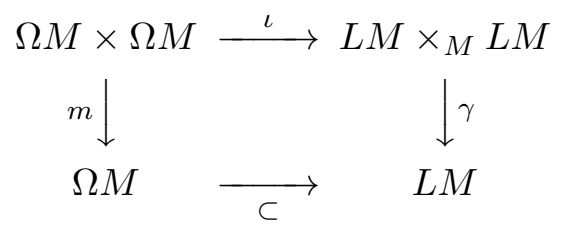

where $m$ is the usual multiplication on the based loop space. Pulling back the virtual bundle $-T M$ over $L M$, and applying the Pontrjagin - Thom construction, we then get a 
commutative diagram of spectra,

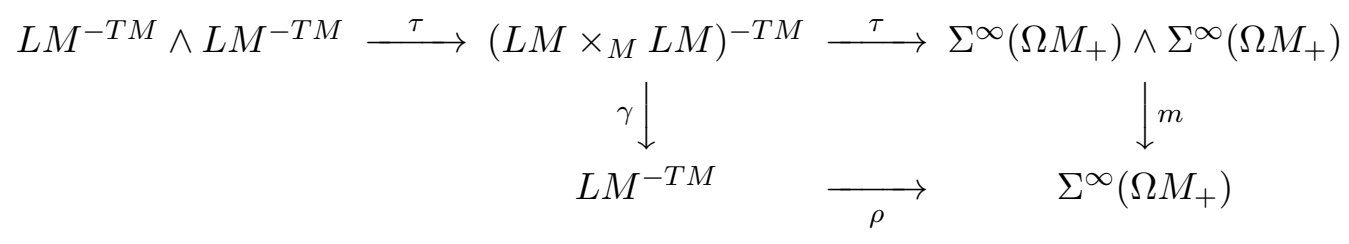

Now as observed above (1.13), the top horizontal composition $\tau \circ \tau$ is equal to $\rho \wedge \rho$ : $L M^{-T M} \wedge L M^{-T M} \rightarrow \Sigma^{\infty}\left(\Omega M_{+}\right) \wedge \Sigma^{\infty}\left(\Omega M_{+}\right)$. Also, $\gamma \circ \tau$ is, by definition, the ring structure $\mu: L M^{-T M} \wedge L M^{-T M} \rightarrow L M^{-T M}$. Thus the following diagram of spectra commutes:

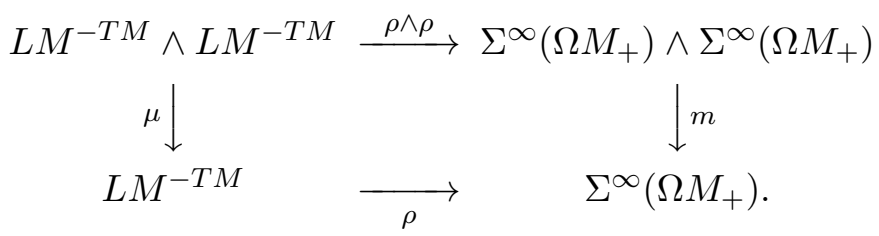

Thus $\rho$ is a map of ring spectra, which completes the proof of theorem 1 . 


\section{THE OPERAD STRUCTURE}

In this section we describe a homotopy theoretic realization of the operad structure on the homology of the loop space found Voronov [6]. The operad involved is the "cactus operad" $\mathcal{C}$ which has the homotopy of the operad of framed little disks in $\mathbb{R}^{2}$ (theorem 2.3 of $[6]$ ). According to Getzler's result [3] this is precisely what is needed to induce the Batalin Vilkovisky algebra structure, and Voronov observed that his operad structure induced the BV structure on $H_{*}(L M)$ described by Chas and Sullivan. Therefore the constructions in this section can be viewed as a homotopy theoretic realization of Chas and Sullivan's $B V$ - algebra structure on $H_{*}(L M)$.

We begin by recalling the definition of cactus operad $\mathcal{C}$. A point in the space $\mathcal{C}_{k}$ is a collection of $k$ oriented, parameterized circles $c_{1}, \cdots, c_{k}$, with radii $r_{i}$ so that $\sum_{i-1}^{k} r_{i}=1$. Each circle has a marked point $x_{i} \in c_{i}$ given by the image under the parameterization of the basepoint $1 \in S^{1}$. Moreover the circles can intersect each other at a finite number of points (vertices) to create a "cactus - type configuration". Strictly speaking this means that the dual graph of this configuration is a tree. That is, the "cactus" (i.e the union of the circles) must be connected and have no "extra loops". (This is the tree condition on the dual graph.) The boundary of the cactus (that is the union of the circles) is also equipped with a fixed basepoint $y_{0}$ together with a choice of which component the basepoint $y_{0}$ lies in. Say $y_{0} \in c_{j_{0}}$. (This choice is only relevant if the basepoint happens to be one of intersection points.) The edges coming into any vertex are also equipped with a cyclic ordering. An ordering of the circle components making up $c$ as well as the vertices within each component is part of the data making up the cactus. The topology of the space of cacti with $k$ - components, $\mathcal{C}_{k}$ is described in [6]. 


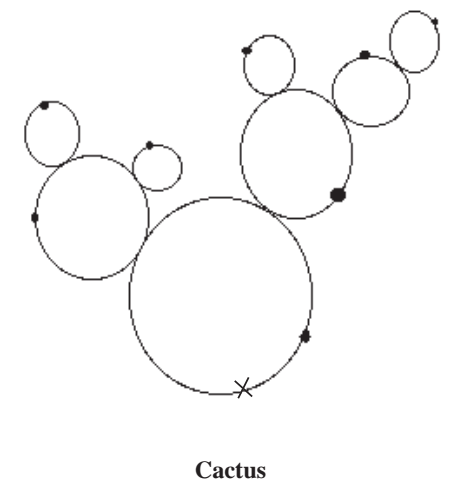

Notice that a cactus (i.e a point in $\mathcal{C}_{k}$ ) comes equipped with a well defined map from the unit circle to the boundary of the cactus. That is, the map begins at the basepoint $y_{0} \in c_{j_{0}}$, then traverses the circle $c_{j 0}$ in the direction of its orientation in a length preserving manner. When a point of intersection with another circle is reached, the loop then traverses that circle, in the direction of its orientation. This path is continued until it eventually arrives back at the original basepoint $y_{0} \in c_{j_{0}}$. Given a cactus $c=\left\langle c_{1}, \cdots, c_{k}\right\rangle \in \mathcal{C}_{k}$ we let $\delta_{c}: S^{1} \rightarrow c_{1} \cup \cdots \cup c_{k}$ be this loop that traverses the boundary of the cactus.

As mentioned above, the result that the cactus operad is homotopy equivalent to the framed little disk operad is a theorem of Voronov [6]. Roughly, the relationship between these operads can seen as follows.

Let $\mathcal{F}_{k}$ be the $k^{t h}$ space of $k$ framed little disks in $\mathbb{R}^{2}$. Recall that there is a homotopy equivalence,

$$
\mathcal{F}_{k} \simeq F\left(\mathbb{R}^{2}, k\right) \times\left(S^{1}\right)^{k}
$$

where $F\left(\mathbb{R}^{2}, k\right)$ is the configuration space of $k$ ordered, distinct points in $\mathbb{R}^{2}$. One can construct a map $\phi_{k}: \mathcal{C}_{k} \rightarrow \mathcal{F}_{k}$ in the following way. View a cactus $c \in \mathcal{C}_{k}$ as a planar graph. From this perspective, the center of each component circle determines a configuration of $k$ points in the plane, $\phi_{k}^{1}(c) \in F\left(\mathbb{R}^{2}, k\right)$. Furthermore, the parameterizations of the component circles determine a point $\phi_{k}^{2}(c) \in\left(S^{1}\right)^{k}$. Then $\phi_{k}$ is defined (up to homotopy) as the 
composition

$$
\phi_{k}: \mathcal{C}_{k} \stackrel{\phi_{k}^{1} \times \phi_{k}^{2}}{\longrightarrow} F\left(\mathbb{R}^{2}, k\right) \times\left(S^{1}\right)^{k} \stackrel{\simeq}{\longrightarrow} \mathcal{F}_{k} .
$$

One can see that the maps $\phi_{k}$ are homotopy equivalences by an inductive argument on the number of components $k$, using natural fibrations $\mathcal{C}_{k} \rightarrow \mathcal{C}_{k-1}$ defined by ignoring the last component circle that does not contain the basepoint, and $\mathcal{F}_{k} \rightarrow \mathcal{F}_{k-1}$ defined by ignoring the last disk. We refer the reader to Voronov's work for a complete proof of this homotopy equivalence.

Notice that $\mathcal{C}_{k}$ has a free action of the symmetric group $\Sigma_{k}$ defined by permuting the ordering of the circles. The operad action

$$
\xi: \mathcal{C}_{k} \times\left(\mathcal{C}_{j_{1}} \times \cdots \times \mathcal{C}_{j_{k}}\right) \rightarrow \mathcal{C}_{j}
$$

where $j=j_{1}+\cdots+j_{k}$ is defined as follows. Let $c \times\left(c^{1}, \cdots, c^{k}\right) \in \mathcal{C}_{k} \times\left(\mathcal{C}_{j_{1}} \times \cdots \times \mathcal{C}_{j_{k}}\right)$. Scale the cactus $c^{1}$ down so that its total radius is the radius $r_{1}$ of the first component $c_{1}$ of $c \in C_{k}$. Similarly, scale each of the cacti $c^{i}$ down so that its total radius is the radius $r_{i}$ of the $i^{\text {th }}$ component $c_{i}$ of $c$. By using the loops $\delta_{c^{i}}$ (scaled down appropriately) we identify the component circles $c_{i}$ with the boundary of the cactis $c^{i}$. This has the effect of replacing the $k$ component circles $c_{1}, \cdots, c_{k}$ making up the cactus $c$, by the $k$ cacti, $c^{1}, \cdots, c^{k}$. This produces a cactus with $j_{1}+\cdots+j_{k}=j$ component circles.

Our goal in this section is to prove theorem 2 as stated in the introduction.

Proof. Given a cactus $c=\left\langle c_{1}, \cdots, c_{k}\right\rangle \in \mathcal{C}_{k}$, define $L_{c} M$ to be the mapping space

$$
L_{c} M=\operatorname{Map}(c, M) \text {. }
$$

This space consists of maps from the union $c_{1} \cup \cdots \cup c_{k} \rightarrow M$. The map from the circle $\delta_{c}: S^{1} \rightarrow c_{1} \cup \cdots \cup c_{k}$ defines a map from $L_{c} M$ to the loop space,

$$
\begin{aligned}
\gamma_{c}: L_{c} M & \rightarrow L M \\
f & \rightarrow f \circ \delta_{c} .
\end{aligned}
$$

Now $L_{c} M$ can also be viewed as the pullback of an evaluation mapping of the product $(L M)^{k}$ defined as follows. For each component of the cactus $c_{i}$, let $y_{1}, \cdots, y_{m_{i}}$ denote the vertices of $c$ that lie on $c_{i} . m_{i}=m_{i}(c)$ is the number of these vertices. Let $m_{c}=$ $m_{1}+\cdots+m_{k}$. We define an evaluation map

$$
e v_{c}:(L M)^{k} \longrightarrow(M)^{m_{c}}
$$

as follows. Let $s_{i}: S^{1} \rightarrow c_{i}$ be the identification of the unit circle with $c_{i}$ obtained by scaling down the unit circle so as to have radius $r_{i}$, and rotating it so the basepoint $1 \in S^{1}$ 
is mapped to the marked point $x_{i} \in c_{i}$. Let $u_{1}, \cdots, u_{m_{i}}$ be the points on the unit circle corresponding to $y_{1}, \cdots y_{m_{i}} \in c_{i}$ under the map $s_{i}$. Define

$$
\begin{aligned}
e v_{c_{i}}: L M & \rightarrow(M)^{m_{i}} \\
\sigma & \rightarrow\left(\sigma\left(u_{1}\right), \cdots, \sigma\left(u_{m_{i}}\right)\right)
\end{aligned}
$$

Now define

$$
e v_{c}=e v_{c_{1}} \times \cdots \times e v_{c_{m_{i}}}: L M \rightarrow(M)^{m_{1}} \times \cdots \times(M)^{m_{i}}=(M)^{m_{c}}
$$

Now let $w_{1}, \cdots, w_{n_{c}} \in c_{1} \cup \cdots \cup c_{k}$ be the ordered collection of vertices in the cactus $c$ (ordered by the ordering of the components $c_{1}, \cdots c_{k}$ and the ordering of the vertices in each component as described above). For each such vertex $w_{i}$, let $\mu_{i}$ be the number of components of the cactus on which $w_{i}$ lies. We think of $\mu_{i}$ as the "multiplicity" of the vertex $w_{i}$. Notice that we have the relation

$$
\sum_{i=1}^{n_{c}} \mu_{i}=m_{c} .
$$

The "tree" condition on the dual of the cactus also imposes the following relation:

$$
m_{c}-n_{c}=k-1
$$

Now consider the diagonal mapping

$$
\Delta_{c}:(M)^{n_{c}} \longrightarrow(M)^{m_{c}}
$$

defined by the composition

$$
\Delta_{c}:(M)^{n_{c}} \stackrel{\Delta_{\mu_{1}} \times \cdots \times \Delta_{\mu_{n_{c}}}}{\longrightarrow}(M)^{\mu_{1}} \times \cdots \times(M)^{\mu_{n_{c}}} \stackrel{=}{\longrightarrow}(M)^{m_{c}}
$$

where $\Delta_{\mu_{i}}: M \rightarrow(M)^{\mu_{i}}$ is the $\mu_{i}$-fold diagonal. Observe that the following is a cartesian pull - back square:

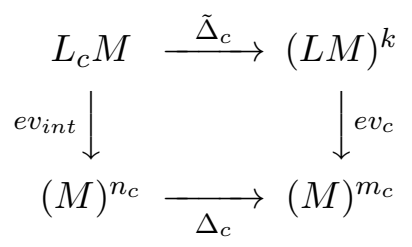

where $e v_{\text {int }}: L_{c} M \rightarrow(M)^{n_{c}}$ evaluates a map $f: c \rightarrow M$ at the $n_{c}$ vertices $w_{1}, \cdots, w_{n_{c}} \in c$.

The normal bundle $\nu\left(\Delta_{c}\right)$ of the diagonal embedding

$$
\Delta_{c}:(M)^{n_{c}} \stackrel{\Delta_{\mu_{1}} \times \cdots \times \Delta_{\mu_{n_{c}}}}{\longrightarrow}(M)^{\mu_{1}} \times \cdots \times(M)^{\mu_{n_{c}}} \stackrel{=}{\longrightarrow}(M)^{m_{c}}
$$


is equal to

$$
\left(\mu_{1}-1\right) T M \times \cdots \times\left(\mu_{n_{c}}-1\right) T M \longrightarrow(M)^{n_{c}},
$$

where $(q) T M$ denotes the $q$ - fold direct sum of $T M$ with itself as a bundle over $M$. By the tubular neighborhood theorem, we have therefore proven the following.

Lemma 4. The image of the embedding $\tilde{\Delta}_{c}: L_{c} M \hookrightarrow(L M)^{k}$ has an open neighborhood homeomorphic to the total space of the pullback ev $v_{\text {int }}^{*}\left(\left(\mu_{1}-1\right) T M \times \cdots \times\left(\mu_{n_{c}}-1\right) T M\right)$.

We now consider these constructions in a parameterized way, by letting $c \in \mathcal{C}_{k}$ vary. Namely, let

$$
L_{k} M=\left\{(c, f): c \in \mathcal{C}_{k} \text { and } f \in L_{c} M\right\}
$$

We then have a map

$$
\begin{aligned}
\tilde{\Delta}_{k}: L_{k} M & \rightarrow \mathcal{C}_{k} \times(L M)^{k} \\
(c, f) & \rightarrow\left(c, \tilde{\Delta}_{c}(f)\right) .
\end{aligned}
$$

We also have a map

$$
\begin{aligned}
\gamma_{k}: L_{k} M & \rightarrow L M \\
(c, f) & \rightarrow \gamma_{c}(f),
\end{aligned}
$$

where $\gamma_{c}$ is as defined in (2.1).

Lemma 1 allows us to identify an open neighborhood of the image of $\tilde{\Delta}_{k}$, as follows. Let $\xi_{k}$ be the $d(k-1)$ dimensional vector bundle over $L_{k} M$ whose fiber over $(c, f)$ is the sum of tangent spaces,

$$
\left(\xi_{k}\right)_{\left.\right|_{(c, f)}}=\bigoplus_{j=1}^{n_{c}}\left(\mu_{j}-1\right) T_{f\left(w_{j}\right)} M
$$

where, as above, $w_{1}, \cdots, w_{n_{c}}$ are the vertices of $c$, and $\mu_{j}=\mu_{j}(c)$ is the multiplicity of the vertex $w_{j}$. It is immediate that $\xi_{k}$ is a well defined vector bundle. Lemma now yields the following.

Lemma 5. The image of the embedding $\tilde{\Delta}_{k}: L_{k} M \rightarrow \mathcal{C}_{k} \times(L M)^{k}$ has an open neighborhood homeomorphic to the total space of $\xi_{k}$.

Notice that this will allow us to perform a Pontrjagin - Thom collapse map

$$
\tau: \mathcal{C}_{k} \times(L M)^{k} \longrightarrow\left(L_{k} M\right)^{\xi_{k}} .
$$


But in order to prove theorem 2, we will need to twist this map by a virtual bundle $\theta_{k}$ over $\mathcal{C}_{k} \times(L M)^{k}$. To define this bundle, first fix an embedding $M \hookrightarrow \mathbb{R}^{d+L}$ with $L>>d$, having normal bundle $\eta$ of dimension $L$. Define a vector bundle $\tilde{\theta}_{k}$ of dimension $L(k-1)$ over $\mathcal{C}_{k} \times(L M)^{k}$ whose fiber at $\left(c ; f_{1}, \cdots f_{k}\right)$ is given by

$$
\left(\tilde{\theta}_{k}\right)_{\left.\right|_{\left(c ; f_{1}, \cdots f_{k}\right)}}=\bigoplus_{j=1}^{n_{c}}\left(\mu_{j}-1\right) \eta_{f\left(x_{j, 1}\right)}
$$

where $x_{j, 1}, \cdots, x_{j, \mu_{j}}$ are the points on $\coprod_{k} S^{1}$ that map to $u_{j}$ under the parameterization $S^{1} \sqcup \cdots \sqcup S^{1} \stackrel{s_{1} \sqcup \cdots \sqcup s_{k}}{\longrightarrow} c_{1} \cup \cdots \cup c_{k}$. (Notice that by the "tree" condition on the cactus, there is at most one of the $x_{j, r}^{\prime} s$ on any one circle in $\coprod_{k} S^{1}$.)

It is clear that these fibers fit together to give a vector bundle $\tilde{\theta}_{k}$ over $\mathcal{C}_{k} \times(L M)^{k}$. Lemma 5 implies the following result.

Lemma 6. Consider the embedding of total spaces of bundles

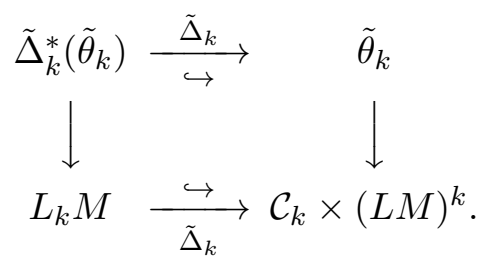

then the image of $\tilde{\Delta}_{k}^{*}\left(\tilde{\theta}_{k}\right)$ in $\tilde{\theta}_{k}$ has an open neighborhood homeomorphic to the total space of the bundle $\tilde{\Delta}_{k}^{*}\left(\tilde{\theta}_{k}\right) \oplus \xi_{k}$ over $L_{k} M$.

This implies we have a Pontrjagin - Thom collapse map,

$$
\tau_{k}:\left(\mathcal{C}_{k} \times(L M)^{k}\right)^{\tilde{\theta}_{k}} \longrightarrow\left(L_{k} M\right)^{\tilde{\Delta}_{k}^{*}\left(\tilde{\theta}_{k}\right) \oplus \xi_{k}} .
$$

Now by definition, $\tilde{\Delta}_{k}^{*}\left(\tilde{\theta}_{k}\right) \oplus \xi_{k}$ is a vector bundle of dimension $(d+L)(k-1)$ whose fiber at $(c, f)$ is the sum of the vector spaces,

$$
\bigoplus_{j=1}^{n_{c}}\left(\mu_{j}-1\right)\left(T_{f\left(w_{j}\right)} M \oplus \eta_{f}\left(w_{j}\right)\right) .
$$

But since $T M \oplus \eta$ is canonically trivial, we have an induced trivialization

$$
\tilde{\Delta}_{k}^{*}\left(\tilde{\theta}_{k}\right) \oplus \xi_{k} \cong L_{k} M \times \mathbb{R}^{(d+L)(k-1)} .
$$

Therefore the Pontrjagin - Thom collapse is a map

$$
\tau_{k}:\left(\mathcal{C}_{k} \times(L M)^{k}\right)^{\tilde{\theta}_{k}} \longrightarrow \Sigma^{(d+L)(k-1)}\left(L_{k} M_{+}\right) .
$$


Let $\theta_{k}$ be the virtual bundle over $L_{k} M$,

$$
\theta_{k}=\tilde{\theta}_{k}-[(d+L)(k-1)]
$$

where $[q]$ refers to the $q$ - dimensional trivial bundle. Notice that $\theta_{k}$ has virtual dimension $-d(k-1)$, and its Thom spectrum is the $-(d+L)(k-1)$ - fold desuspension of the Thom spectrum of $\tilde{\theta}_{k}$. We then define $\mu_{k}$ to be the composition,

$$
\mu_{k}:\left(\mathcal{C}_{k} \times(L M)^{k}\right)^{\theta_{k}} \stackrel{\Sigma^{-(d+L)(k-1)} \tau_{k}}{\longrightarrow} L_{k} M \stackrel{\gamma_{k}}{\longrightarrow} L M .
$$

Now recall from $([6])$ that the cactus operad structure on $H_{*}(L M)$ is given by the composition

$$
H_{*}\left(\mathcal{C}_{k} \times(L M)^{k}\right) \stackrel{\left(\tilde{\Delta}_{k}\right) !}{\longrightarrow} H_{*}\left(L_{k} M\right) \stackrel{\left(\gamma_{k}\right)_{*}}{\longrightarrow} H_{*}(L M)
$$

where $\left(\tilde{\Delta}_{k}\right)$ ! is the push -forward map. (Note that our notation is different than that used in [6].) The theorem now follows from the well known fact that the Pontrjagin - Thom collapse map realizes the push -forward map in homology. 
3. A Cosimplicial description of $L M$ And $L M^{-T M}$ And a proof of Theorem 3

In this section we describe a cosimplicial model for the spectrum $L M^{-T M}$. We then describe the ring spectrum structure simplicially. This cosimplicial model will then give a natural way of relating the singular chains $C_{*}\left(L M^{-T M}\right)$ to the Hochschild cochain complex $C H^{*}\left(C^{*}(M), C^{*}(M)\right)$, and in particular relate the simplicial model for the ring structure of $L M^{-T M}$ to the cup product structure in this cochain complex. This will allow us to prove theorem 3 .

We begin by reviewing the cosimplicial model of the loop space $L X$ for any space $X$, coming from a simplicial decomposition of the circle $S^{1}$. We refer the reader to [4] for details.

Let $S_{*}^{1}$ be the simplicial set decomposition of the circle which has one zero simplex and one nondegenerate one simplex. In this decomposition there are $n+1 n$ - simplices, all of which are degenerate for $n>1$. We write this as $S_{n}^{1}=\{n+1\}$. Now given any space $X$, there is a resulting cosimplicial model for the free loop space, $L X$, which we call $\mathbb{X}_{*}$. The $n$ - simplices of $\mathbb{X} *$ are given by maps

$$
\mathbb{X}_{n}=\operatorname{Map}\left(S_{n}^{1}, X\right)=\operatorname{Map}(\{n+1\}, X)=X^{n+1} .
$$

The coface and codegeneracy maps of $\mathbb{X}_{*}$ are dual to the face and degeneracy maps of $S_{*}^{1}$. They are given by the formulas

$$
\begin{aligned}
\delta_{i}\left(x_{0}, \cdots, x_{n-1}\right) & =\left(x_{0}, \cdots, x_{i-1}, x_{i}, x_{i}, x_{i+1}, \cdots, x_{n-1}\right), \quad 0 \leq i \leq n-1 \\
\delta_{n}\left(x_{0}, \cdots, x_{n-1}\right) & =\left(x_{0}, x_{1}, \cdots, x_{n-1}, x_{0}\right) \\
\sigma_{i}\left(x_{0}, \cdots, x_{n+1}\right) & =\left(x_{0}, \cdots, x_{i}, x_{i+2}, \cdots, x_{n+1}\right), \quad 0 \leq i \leq n
\end{aligned}
$$

Since the geometric realization of $S_{*}^{1}$ is homeomorphic to the circle,

$$
S^{1} \cong\left|S_{*}^{1}\right|,
$$

the "total complex" or geometric corealization of $\mathbb{X}_{*}$ is homeomorphic to the loop space,

$$
L X \cong \operatorname{Tot}\left(\mathbb{X}_{*}\right)
$$

This was studied in detail by the second author in [4], and in particular the following interpretation of this result was given. For each $k$, let $\Delta^{k}$ be the standard $k$ - simplex:

$$
\Delta^{k}=\left\{\left(x_{1}, \cdots, x_{k}\right): 0 \leq x_{1} \leq x_{2} \leq \cdots \leq x_{k} \leq 1\right\} .
$$


Consider the maps

$$
\begin{aligned}
f_{k}: \Delta^{k} \times L X & \longrightarrow X^{k+1} \\
\left(x_{1}, \cdots, x_{k}\right) \times \gamma & \rightarrow\left(\gamma(0), \gamma\left(x_{1}\right), \cdots, \gamma\left(x_{k}\right)\right) .
\end{aligned}
$$

Let $\bar{f}_{k}: L X \rightarrow \operatorname{Map}\left(\Delta^{k}, X^{k+1}\right)$ be the adjoint of $f_{k}$. Then the following was proven in 沟.

Theorem 7. Let $X$ be any space, and let $f: L X \longrightarrow \prod_{k \geq 0} M a p\left(\Delta^{k}, X^{k+1}\right)$ be the product of the maps $\bar{f}_{k}$. Then $f$ is a homeomorphism onto its image. Furthermore, the image consists of sequences of maps $\left\{\phi_{k}\right\}$ which commute with the coface and codegeneracy operators, which is the total space, $\operatorname{Tot}\left(\mathbb{X}_{*}\right)$.

By applying singular cochains to the maps $f_{k}$, one obtains maps

$$
f_{k}^{*}: C^{*}(X)^{\otimes k+1} \rightarrow C^{*-k}(L X) .
$$

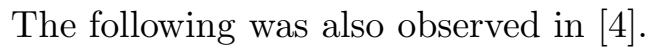

Theorem 8. For any space $X$, the homomorphisms $f_{k}^{*}: C^{*}(X)^{\otimes k+1} \rightarrow C^{*-k}(L X)$ fit together to define a chain map from the Hochschild complex of the cochains of $X$ to the cochains of the free loop space,

$$
f^{*}: C H_{*}\left(C^{*}(X)\right) \rightarrow C^{*}(L(X))
$$

which is a chain homotopy equivalence when $X$ is simply connected. Hence it induces an isomorphism in homology

$$
f^{*}: H H_{*}\left(C^{*}(X)\right) \stackrel{\cong}{\longrightarrow} H^{*}(L(X)) .
$$

Remark. Let us clarify some notation. Given an algebra (or differential graded algebra) $A$, the the Hochschild complex of $A, C H_{*}(A)$ is a complex of the form

$$
\cdots \stackrel{b}{\longrightarrow} A^{\otimes n+2} \stackrel{b}{\longrightarrow} A^{\otimes n+1} \stackrel{b}{\longrightarrow} \cdots \stackrel{b}{\longrightarrow} A \otimes A \stackrel{b}{\longrightarrow} A .
$$

The homology of this algebra is denoted $H H_{*}(A)$. More generally if $M$ is a bimodule over $A$, we denote by $C H_{*}(A ; M)$ the Hochschild complex of the form

$$
\cdots \stackrel{b}{\longrightarrow} A^{\otimes n+1} \otimes M \stackrel{b}{\longrightarrow} A^{\otimes n} \otimes M \stackrel{b}{\longrightarrow} \cdots \stackrel{b}{\longrightarrow} A \otimes M \stackrel{b}{\longrightarrow} M .
$$


The homology of this complex is denoted $H H_{*}(A ; M)$. So in particular if $M=A$ we see that $H H_{*}(A ; A)=H H_{*}(A)$. Dually, we denote by $C H^{*}(A ; M)$ the Hochschild cochain complex of the form

$$
M \stackrel{b^{*}}{\longrightarrow} \operatorname{Hom}(A ; M) \stackrel{b^{*}}{\longrightarrow} \cdots \stackrel{b^{*}}{\longrightarrow} \operatorname{Hom}\left(A^{\otimes n} ; M\right) \stackrel{b^{*}}{\longrightarrow} \operatorname{Hom}\left(A^{\otimes n+1} ; M\right) \stackrel{b^{*}}{\longrightarrow} \cdots
$$

Its cohomology is denoted $H H^{*}(A ; M)$. By dualizing theorem 0 we obtain the following.

Corollary 9. For any simply connected space $X$, there is a chain homotopy equivalence from the singular chains of the loop space to the Hochschild cochain complex

$$
f_{*}: C_{*}(L X) \rightarrow C H^{*}\left(C^{*}(X) ; C_{*}(X)\right)
$$

and so an isomorphism in homology,

$$
f_{*}: H_{*}(L X) \stackrel{\cong}{\longrightarrow} H H^{*}\left(C^{*}(X) ; C_{*}(X)\right) .
$$

Notice that the cochain complex $C H^{*}\left(C^{*}(X) ; C_{*}(X)\right)$ does not in general have a natural product structure. This is because the coefficients, $C_{*}(X)$, is not in general a ring. Notice however that the Hochschild complex $C H^{*}\left(C^{*}(X), C^{*}(X)\right)$ does in fact have a cup product coming from the algebra structure of $C^{*}(X)$. Of course when $X$ is a closed, oriented manifold of dimension $d$, Poincare duality gives a chain homotopy equivalence, $C_{*}(X) \cong C^{d-*}(X)$, and so the cochain complex $C H^{*}\left(C^{*}(X) ; C_{*}(X)\right)$ inherits an algebra structure. Therefore by the above corollary, $H_{*}(L X)$ inherits an algebra structure in this case. We will see that this indeed realizes the Chas - Sullivan product. We will show this by showing that when $M$ is a closed $d$ - manifold, the Thom spectrum $L M^{-T M}$ inherits a cosimplicial structure for which the analogue of theorem 8 will yield a natural chain homotopy equivalence $C_{*}\left(L M^{-T M}\right) \cong$ $C H^{*}\left(C^{*}(M), C^{*}(M)\right)$.

To begin, notice that by the definitions 3.2, the following diagrams commute:

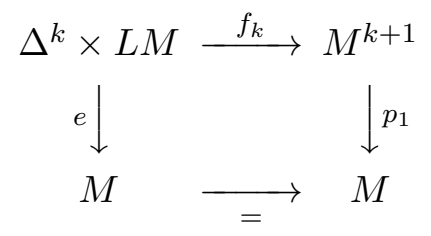

where the left hand vertical map is the evaluation, $e\left(\left(t_{1}, \cdots, t_{k}\right) ; \gamma\right)=\gamma(0)$, and the right hand vertical map is the projection onto the first coordinate. Pulling back the virtual bundle $-T(M)$ defines a map of virtual bundles

$$
\left(f_{k}\right)_{*}: e^{*}(-T M) \longrightarrow p_{1}^{*}(-T M),
$$


and therefore maps of Thom spectra, (which by abuse of notation we still call $f_{k}$ )

$$
f_{k}:\left(\Delta_{k}\right)_{+} \wedge L M^{-T M} \longrightarrow M^{-T M} \wedge\left(M^{k}\right)_{+} .
$$

By taking adjoints, we get a map of spectra,

$$
f: L M^{-T M} \stackrel{\prod_{k} f_{k}}{\longrightarrow} \prod_{k} M a p\left(\left(\Delta_{k}\right)_{+} ; M^{-T M} \wedge\left(M^{k}\right)_{+}\right)
$$

where on the right hand side the mapping spaces are maps of unital spectra. This map is just the induced map of Thom spectra of the map $f: L X \longrightarrow \prod_{k \geq 0} \operatorname{Map}\left(\Delta^{k}, X^{k+1}\right)$ described in theorem 0 . The following result is induced by theorem 0 by passing to Thom spectra.

Let $\left(\mathbb{L}_{M}\right)_{*}$ be the cosimplicial spectrum defined to be the cosimplicial Thom spectrum of the cosimplicial virtual bundle $-T M$. That is, the virtual bundle over the $k$ simplices $M^{k+1}$ is $p_{1}^{*}(-T M)$. Said more explicitly, $\left(\mathbb{L}_{M}\right)_{*}$ is the cosimplicial spectrum whose $k$ - simplices are the spectrum

$$
\left(\mathbb{L}_{M}\right)_{k}=M^{-T M} \wedge\left(M^{k}\right)_{+} .
$$

To describe the coface and codegeneracy maps, consider the maps

$$
\mu_{L}: M^{-T M} \rightarrow M_{+} \wedge M^{-T M} \quad \text { and } \quad \mu_{R}: M^{-T M} \rightarrow M^{-T M} \wedge M_{+}
$$

of Thom spectra induced by the diagonal map $\Delta: M \rightarrow M \times M . \mu_{L}$ and $\mu_{R}$ are the maps of Thom spectra induced by the maps of virtual bundles $\Delta_{*}:-T M \rightarrow p_{L}^{*}(-T M)$ and $\Delta_{*}:-T M \rightarrow p_{R}^{*}(-T M)$, where $p_{L}$ and $p_{R}$ are the projection maps $M \times M \rightarrow M$ onto the left and right coordinates respectively. We then have the following formulas for the coface and codegeneracy maps:

$$
\begin{aligned}
\delta_{0}\left(u ; x_{1}, \cdots, x_{k-1}\right) & =\left(v_{R} ; y_{R}, x_{1}, \cdots, x_{k-1}\right) \\
\delta_{i}\left(u ; x_{1}, \cdots, x_{k-1}\right) & =\left(u ; x_{1}, \cdots, x_{i-1}, x_{i}, x_{i}, x_{i+1}, \cdots, x_{k-1}\right), \quad 1 \leq i \leq k-1 \\
\delta_{k}\left(u ; x_{1}, \cdots, x_{k-1}\right) & =\left(v_{L} ; x_{1}, \cdots, x_{k-1}, y_{L}\right),
\end{aligned}
$$

where $\mu_{R}(u)=\left(v_{R}, y_{R}\right), \mu_{L}(u)=\left(y_{L}, v_{L}\right)$ and

$$
\sigma_{i}\left(u ; x_{1}, \cdots, x_{k+1}\right)=\left(u ; x_{1}, \cdots, x_{i}, x_{i+2}, \cdots, x_{k+1}\right), \quad 0 \leq i \leq k
$$

The following result is simply the application of the Thom spectrum functor for the virtual bundle $-T M$ to theorem 0 . 
Theorem 10. Let $M$ be any closed, $d$ - dimensional manifold, and let

$$
f: L M^{-T M} \stackrel{\prod_{k} f_{k}}{\longrightarrow} \prod_{k} M a p\left(\left(\Delta_{k}\right)_{+} ; M^{-T M} \wedge\left(M^{k}\right)_{+}\right)
$$

be the product of the maps of spectra $f_{k}$, as defined above (3.马). Then $f$ is a homeomorphism onto its image. Furthermore, the image consists of sequences of maps $\left\{\phi_{k}\right\}$ which commute with the coface and codegeneracy operators.

We denote the space of sequences of maps referred to in this theorem $\operatorname{Map}_{\Delta^{*}}\left(\Delta^{*}, M^{-T M} \wedge\right.$ $\left.\left(M^{k}\right)_{+}\right)$. This is the total space of the cosimplicial spectrum $\operatorname{Tot}\left(\left(\mathbb{L}_{M}\right)_{*}\right)$.

Notice that the $S$ - duality between $M_{+}$and $M^{-T M}$ defines a chain homotopy equivalence between the cochains $C^{*}\left(M^{-T M}\right)$ (defined to be the appropriate desuspension of the cochains of the Thom space of the normal bundle of a fixed embedding $M \hookrightarrow \mathbb{R}^{N}$ ) and the chains of the manifold

$$
C^{*}\left(M^{-T M}\right) \cong C_{-*}\left(M_{+}\right)
$$

The maps $f_{k}:\left(\Delta_{k}\right)_{+} \wedge L M^{-T M} \longrightarrow M^{-T M} \wedge\left(M^{k}\right)_{+}$then define maps of cochains,

$$
f_{k}^{*}: C_{-*}(M) \otimes C^{*}(M)^{\otimes k} \cong C^{*}\left(M^{-T M} \wedge\left(M^{k}\right)_{+}\right) \longrightarrow C^{*-k}\left(L M^{-T M}\right) .
$$

Taking the dual we get a map of chain complexes

$$
\begin{aligned}
\left(f_{k}\right)_{*}: C_{*-k}\left(L M^{-T M}\right) & \longrightarrow \operatorname{Hom}\left(C^{*}(M)^{\otimes k} \otimes C_{-*}(M) ; \mathbb{Z}\right) \\
& \cong \operatorname{Hom}\left(C^{*}(M)^{\otimes k} ; C^{*}(M)\right) \\
& =C H^{k}\left(C^{*}(M) ; C^{*}(M)\right)
\end{aligned}
$$

The following is then a consequence of corollary 9, by passing to Thom spectra.

Corollary 11. For any closed manifold $M$, the chain maps $\left(f_{k}\right)_{*}$ fit together to define a chain homotopy equivalence from the chains of Thom spectrum $L M^{-T M}$ to the Hochschild cochain complex

$$
f_{*}: C_{*}\left(L M^{-T M}\right) \rightarrow C H^{*}\left(C^{*}(M) ; C^{*}(M)\right)
$$

and so an isomorphism in homology,

$$
f_{*}: H_{*}\left(L M^{-T M}\right) \stackrel{\cong}{\longrightarrow} H H^{*}\left(C^{*}(M) ; C^{*}(M)\right)
$$


As mentioned in the introduction, the Hochschild cochain complex $C H^{*}\left(C^{*}(M) ; C^{*}(M)\right)$ has a cup product structure. Namely, for any algebra $A$, if

$$
\phi \in C H^{k}(A ; A)=H o m\left(A^{\otimes k} ; A\right) \quad \text { and } \quad \psi \in C H^{r}(A ; A)=H o m\left(A^{\otimes r} ; A\right),
$$

then

$$
\phi \cup \psi \in C H^{k+r}(A ; A)=H o m\left(A^{\otimes k+r} ; A\right)
$$

is defined by

$$
\phi \cup \psi\left(a_{1} \otimes \cdots \otimes a_{k} \otimes a_{k+1} \otimes \cdots \otimes a_{k+r}\right)=\phi\left(a_{1} \otimes \cdots \otimes a_{k}\right) \psi\left(a_{k+1} \otimes \cdots \otimes a_{k+r}\right) .
$$

For $A=C^{*}(M)$ (where the algebra stucture is the cup product in $C^{*}(M)$ ), by taking adjoints, we can think of this as a pairing

$$
\begin{aligned}
\cup:\left(C_{*}(M)^{\otimes k} \otimes C^{*}(M)\right) \otimes\left(C_{*}(M)^{\otimes r} \otimes C^{*}(M)\right) & \longrightarrow C_{*}(M)^{\otimes k+r} \otimes C^{*}(M) \\
\left(\alpha_{1} \otimes \cdots \otimes \alpha_{k} \otimes \theta\right) \otimes\left(\beta_{1} \otimes \cdots \otimes \beta_{r} \otimes \rho\right) & \longrightarrow \alpha_{1} \otimes \cdots \otimes \alpha_{k} \otimes \beta_{1} \otimes \cdots \otimes \beta_{r} \otimes \theta \cup \rho .
\end{aligned}
$$

Now recall that by $S$ - duality, there is a ring spectrum structure

$$
\Delta^{*}: M^{-T M} \wedge M^{-T M} \longrightarrow M^{-T M}
$$

dual to the diagonal map $\Delta: M \rightarrow M \times M$. Passing to chains, $\Delta^{*}: C_{*}\left(M^{-T M}\right) \otimes$ $C_{*}\left(M^{-T M}\right) \rightarrow C_{*}\left(M^{-T M}\right)$, is, with respect to the duality identification $C_{*}\left(M^{-T M}\right) \cong$ $C^{*}(M)$, the cup product on cochains

$$
\Delta^{*}=\cup: C^{*}(M) \otimes C^{*}(M) \rightarrow C^{*}(M) .
$$

Thus formula (3.5) for the cup product in Hochschild cochains is therefore realized by the map

$$
\begin{aligned}
\tilde{\mu}_{k, r}:\left[M^{-T M} \wedge\left(M^{k}\right)_{+}\right] \wedge\left[M^{-T M} \wedge\left(M^{r}\right)_{+}\right] & \longrightarrow M^{-T M} \wedge\left(M^{k+r}\right)_{+} \\
& \left(u ; x_{1}, \cdots, x_{k}\right) \wedge\left(v ; y_{1}, \cdots, y_{r}\right) \longrightarrow\left(\Delta^{*}(u, v) ; x_{1}, \cdots, x_{k}, y_{1}, \cdots, y_{r}\right)
\end{aligned}
$$

The maps $\tilde{\mu}_{k, r}$ define maps of the simplices

$$
\tilde{\mu}_{k, r}:\left(\mathbb{L}_{M}\right)_{k} \wedge\left(\mathbb{L}_{M}\right)_{r} \rightarrow\left(\mathbb{L}_{M}\right)_{k+r}
$$

and it is straight forward to check that these maps preserve the coface and codegeneracy operators, and induce a map of total spectra,

$$
\tilde{\mu}: \operatorname{Tot}\left(\left(\mathbb{L}_{M}\right)_{*}\right) \wedge \operatorname{Tot}\left(\left(\mathbb{L}_{M}\right)_{*}\right) \longrightarrow \operatorname{Tot}\left(\left(\mathbb{L}_{M}\right)_{*}\right) \text {. }
$$

Notice in particular that this pairing is $A_{\infty}$ associative. This proves the following. 
Theorem 12. Using the homeomorphism $f: L M^{-T M} \cong \operatorname{Tot}\left(\left(\mathbb{L}_{M}\right)_{*}\right)$ of theorem 10 , $L M^{-T M}$ inherits the structure of an $A_{\infty}$ ring spectrum,

$$
\tilde{\mu}: L M^{-T M} \wedge L M^{-T M} \longrightarrow L M^{-T M}
$$

which is compatible with the cup product in Hochschild cohomology. That is, with respect to the chain homotopy equivalence

$$
f_{*}: C_{*}\left(L M^{-T M}\right) \rightarrow C H^{*}\left(C^{*}(M) ; C^{*}(M)\right)
$$

of corollary 11, the following diagram of chain complexes commutes:

$$
\begin{array}{ccc}
C_{*}\left(L M^{-T M}\right) \otimes C_{*}\left(L M^{-T M}\right) & \stackrel{\tilde{\mu}}{\longrightarrow} & C_{*}\left(L M^{-T M}\right) \\
f_{*} \otimes f_{*} \downarrow & \cong & \cong \downarrow f_{*} \\
C H^{*}\left(C^{*}(M) ; C^{*}(M)\right) \otimes C H^{*}\left(C^{*}(M) ; C^{*}(M)\right) & \longrightarrow & \longrightarrow H^{*}\left(C^{*}(M) ; C^{*}(M)\right) .
\end{array}
$$

In view of theorems 10, 11, and 12, theorem 3 will therefore be proven once we prove the following.

Theorem 13. Let $\mu: L M^{-T M} \wedge L M^{-T M} \rightarrow L M^{-T M}$ be the ring spectrum structure defined in section 1. Then the structures $\mu$ and $\tilde{\mu}: \operatorname{Tot}\left(\left(\mathbb{L}_{M}\right)_{*}\right) \wedge \operatorname{Tot}\left(\left(\mathbb{L}_{M}\right)_{*}\right) \rightarrow \operatorname{Tot}\left(\left(\mathbb{L}_{M}\right)_{*}\right)$ are compatible in the sense that the following diagram homotopy commutes:

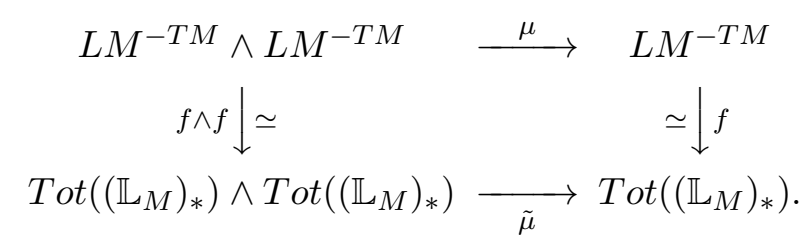

Proof. Let $w_{k, r}: \Delta^{k+r} \rightarrow \Delta^{k} \times \Delta^{r}$ be the Alexander - Whitney diagonal map. That is, for $\left(x_{1}, \cdots, x_{k+r}\right) \in \Delta^{k+r}$, then $w_{k, r}\left(x_{1}, \cdots, x_{k+r}\right)=\left(x_{1}, \cdots, x_{k}\right) \times\left(x_{k+1}, \cdots, x_{k+r}\right) \in$ $\Delta^{k} \times \Delta^{r}$. By the definition of the cosimplicial structure of $\left(\mathbb{L}_{M}\right)_{*}$, to prove the theorem it suffices to prove that the following diagrams of spectra commute: 
(a)

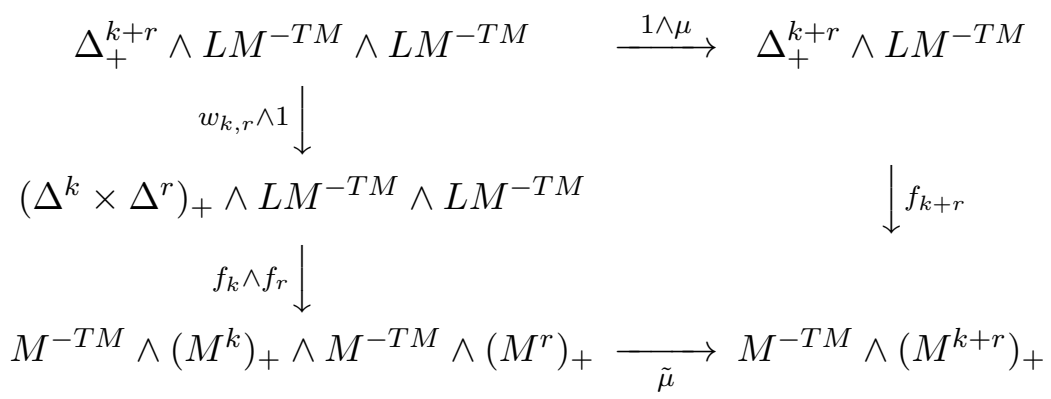

We verify the commutativity of these diagrams in several steps. First observe that the maps

$$
f_{k} \times f_{r}: \Delta^{k} \times \Delta^{r} \times L M \times L M \rightarrow M^{k+1} \times M^{r+1}
$$

restrict to $\Delta^{k} \times \Delta^{r} \times L M \times{ }_{M} L M$ to define a map $f_{k, r}$ whose image is in $M \times M^{k} \times M^{r}$ making the following diagram commute:

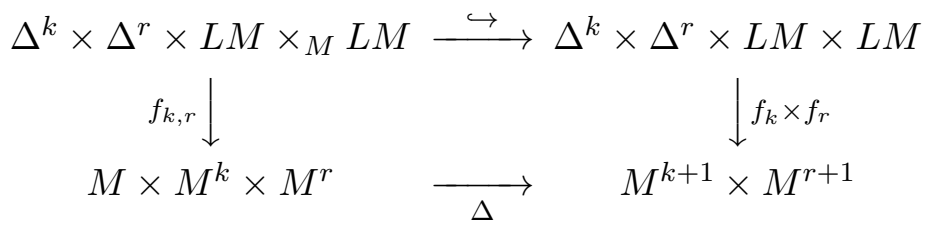

where the bottom horizontal map is the diagonal map:

$$
\Delta\left(m \times\left(x_{1}, \cdots, x_{k}\right) \times\left(y_{1}, \cdots, y_{r}\right)\right)=\left(m, x_{1}, \cdots, x_{k}\right) \times\left(m, y_{1}, \cdots, y_{r}\right) .
$$

By the naturality of the Pontrjagin - Thom construction, we therefore have a commutative diagram of spectra

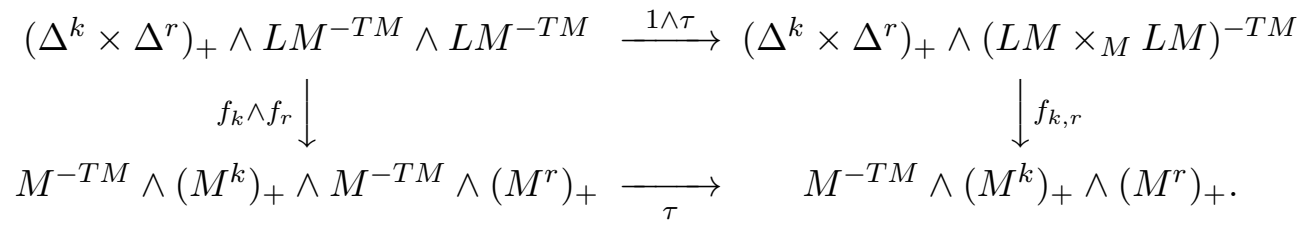

Notice further that by the definition of the maps $f_{n}, f_{k, r}$ and the loop composition $\gamma$ : $L M \times_{M} L M \rightarrow L M$ defined in the last section, the following diagram commutes: 


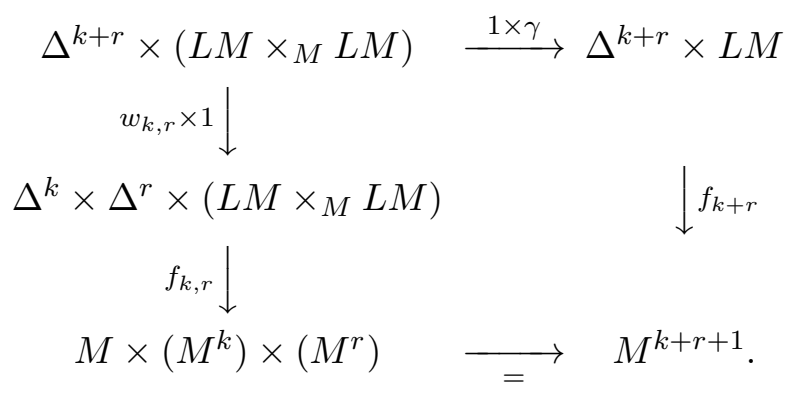

Passing to Thom spectra this yields the following commutative diagram:

(c)

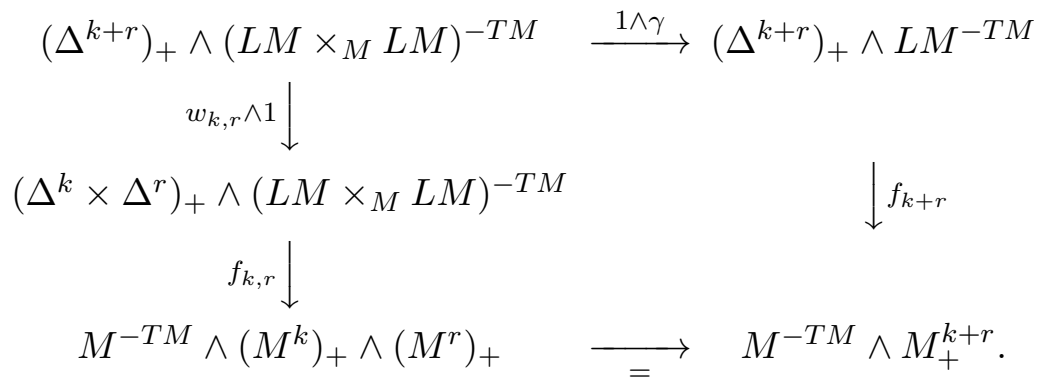

Now consider the following diagram of spectra:

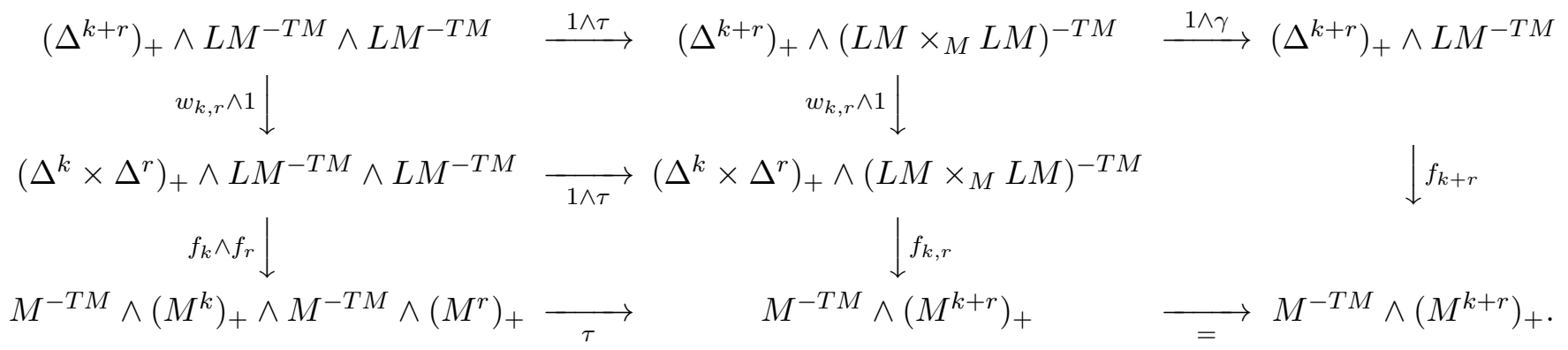

Now the top left square in this diagram clearly commutes. The bottom left diagram is diagram (b) above, and so it commutes. The right hand rectangle is diagram (c) above, so it commutes. Therefore the outside of this diagram commutes. Now the top horizontal composition is, by definition the map

$$
1 \wedge \mu:\left(\Delta^{k+r}\right)_{+} \wedge L M^{-T M} \wedge L M^{-T M} \longrightarrow\left(\Delta^{k+r}\right)_{+} \wedge L M^{-T M} .
$$

The bottom horizontal map is seen to be

$$
\tilde{\mu}: M^{-T M} \wedge\left(M^{k}\right)_{+} \wedge M^{-T M} \wedge\left(M^{r}\right)_{+} \longrightarrow M^{-T M} \wedge\left(M^{k+r}\right)_{+}
$$


by recalling that the ring multiplication $\Delta^{*}: M^{-T M} \wedge M^{-T M} \rightarrow M^{-T M}$ is the Pontrjagin

- Thom map $\tau: M^{-T M} \wedge M^{-T M} \rightarrow M^{-T M}$ applied to the diagonal embedding $\Delta: M \hookrightarrow$ $M \times M$.

With these identifications, the outside of this diagram is then diagram (a) above. As observed earlier, the commutativity of diagram (a) proves this theorem, and this completes the proof of theorem 2 .

\section{REFERENCES}

[1] M.F. Atiyah, Thom complexes, Proc. London Math. Soc. (3), no. 11 (1961), 291-310.

[2] M. Chas and D. Sullivan, String Topology, preprint: math.GT/9911159, (1999).

[3] E. Getzler, Batalin-Vilkovisky algebras and two-dimensional topological field theories., Comm. Math. Phys. 159 no. 2,, (1994), 265-285.

[4] J.D.S Jones, Cyclic homology and equivariant homology Inventionnes Math. 87, no. 2, (1987), 403-423.

[5] T. Tradler, Ph.D thesis, CUNY, to appear.

[6] A.A. Voronov, Notes on Universal Algebra, preprint QA/0111009, t0 o appear in Proc. of Conf. on Graphs in honor of D. Sullivan, June, 2001.

Dept. of Mathematics, Stanford University, Stanford, California 94305

E-mail address, Cohen: ralph@math.stanford.edu

Department of Mathematics, University of Warwick, Coventry, CV4 7AL England

E-mail address, Jones: jdsj@maths.warwick.ac.uk 